Proceedings of SALT 21: 495-514, 2011

\title{
Unconditional concealed questions and Heim's ambiguity*
}

\author{
Ilaria Frana \\ University of Göttingen
}

\author{
Kyle Rawlins \\ Johns Hopkins University
}

\begin{abstract}
In this paper, we investigate Concealed Questions (CQs) in the context of headed unconditionals. We observe that although CQs are licensed in unconditionals, the distribution of readings involved in Heim's Ambiguity (Heim 1979) does not match that found in attitude contexts. Furthermore, the distribution of readings varies by verb class (epistemic vs. communication verbs). We propose that unconditional concealed questions involve questions derived from the denotation of the DP via a specially devised type-shifter, and show how this can block the unwanted readings in exactly the right cases. Heim's ambiguity, we suggest, is not a unitary phenomenon, and a hybrid concept/question-based account is necessary to derive the right readings in the right contexts.
\end{abstract}

Keywords: Attitudes, concealed questions, Heim's ambiguity, unconditionals, verba dicendi

\section{Introduction}

Analyses of Concealed Questions (CQs) have been largely tied to the semantics of attitude ascriptions (Heim 1979; Romero 2005; Frana 2006; Nathan 2006; Schwager 2008; Roelofsen \& Aloni 2008; Frana 2010 a.o.). In this paper, we contribute to disentangling CQs from attitudes by introducing new data involving (non-attitudinal) headed unconditionals. Some examples are provided in (1-2).

(1) Regardless of the temperature outside, I am going for a run.

$$
\Rightarrow \text { Regardless of what the temperature outside is, ... }
$$

(2) Regardless of the computer that Clara buys, she will be able to install $\mathrm{LT}_{\mathrm{E}} \mathrm{X}$. $\Rightarrow$ Regardless of what (kind of) computer Clara buys, ...

In the unconditional sentences above, the italicized definite DPs are not used to pick out a particular entity in the world (a temperature value, or a computer type). Rather, their meaning is better reflected by an embedded question paraphrase,

* For discussion of this work we are grateful to Angelika Kratzer, Marcin Morzycki, Paul Portner, Magdalena Kaufmann, Anne-Michelle Tessier, Alexis Wellwood, Colin Wilson, the members of the JHU Semantics Lab, and audiences at SALT 21 and University of Goettingen; we are also grateful to anonymous SALT and WCCFL reviewers.

C2011 Ilaria Frana \& Kyle Rawlins 
hence the name Concealed Questions (CQs). Beyond simply understanding how the meaning of a CQ should be derived in unconditionals like (1) and (2), this paper focuses on a particular type of unconditional CQ, which in attitude context triggers what is known as Heim's ambiguity. Heim's ambiguity has played a central role in the discussion of CQs in the literature (Heim 1979; Romero 2005; Schwager 2008; Roelofsen \& Aloni 2008; Frana 2010). In this paper, we contribute to the ongoing debate on which analysis is best suited to cover the empirical domain by discussing novel data which will shed light on the nature of Heim's ambiguity and CQs in general. The central puzzle is what we call the what-value reading gap, where one of the readings in Heim's ambiguity is unexpectedly missing in unconditionals.

Heim's ambiguity can be illustrated by the example in (3). Assume a context in which the prices of several new Apple products are under discussion. Suppose further that Miles knows only one of these prices, i.e. he knows the price of the new iPhone. In this scenario, (3) can mean that both Clara and Miles know how much the new iPhone costs, i.e. they both know the answer to the same price-question: "What is the price (value) of the new iPhone?" (Often referred to as an 'A' reading, we will call this the what-value reading). However, (3) has an additional reading according to which Clara knows the answer to a question about Miles' knowledge - she can answer the question "What price (question) does Miles know (the answer to)?" (the B or in our terms, which-concept reading). In this case, Clara does not need to know the answer to the price-question "What is the price of the new iPhone?".

Clara knows the price that Miles knows.

(Heim 1979)

What-value/A: Clara knows the same price as Miles, e.g. the price of the iPhone.

Which-concept/B: Clara knows which price Miles knows.
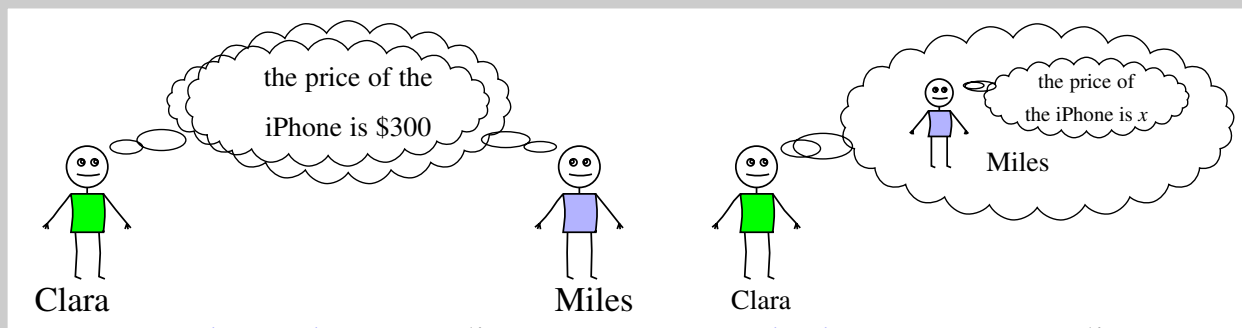

What value/A reading

Which concept/B reading

We will use the term nested CQs to refer to the complex CQs that can lead to Heim's ambiguity. The distinctive feature of nested CQs is that the head noun is modified by a relative clause which contains a CQ-embedding predicate. All the other CQs, with or without relative clauses, will be referred to as simple CQs.

Nested CQs in unconditionals show an interesting contrast between epistemic verbs (know, find out, discover, etc.) and verbs of communication (tell, announce, 
etc.). ${ }^{1}$ As shown in the examples below, nested CQs in unconditionals cannot have what value readings when the verb (inside the RC) is epistemic (5a). However, what value readings are available with communication verbs (5b). Which concept readings, on the other hand, are available with both epistemic and communication verbs, as shown by (6a) and (6b).

(5) Scenario 1 (single product/what-value): Apple is announcing the price of a single product tomorrow - the new iPhone. Miles has inside information and knows in advance what this price is. We are discussing whether to ask Miles about this price.

a. \# Regardless of the price that Miles knows, I won't buy one, so what's the point? $\quad \Rightarrow$ \# Regardless of what the price of the new iPhone is, ...

b. Regardless of the price that Miles tells us, I won't buy one, so what's the point? $\quad \Rightarrow$ Regardless of what the price of the new iPhone is, ...

(6) Scenario 2 (multiple products/which-concept): Apple is announcing the price of several products tomorrow. Miles has some inside information and knows just one of these prices, but we don't know which. We are discussing whether to ask Miles about his inside information.

a. Regardless of the price that Miles knows, we should still watch to find out all the rest, so why bother?

$\Rightarrow$ Regardless of which product Miles knows the price of, ...

b. Regardless of the price that Miles tells us, we should still watch.

$\Rightarrow$ Regardless of which product he tells us the price of, ...

The unconditional data above is puzzling for at least two reasons. First, it reveals a surprising construction asymmetry: the nested CQ (the price that Miles knows) generates an ambiguity in attitude contexts (3), but not in unconditionals, where the what-value reading is not available (5a). Second, within unconditionals we find a puzzling asymmetry with respect to the predicate inside the nested CQ (we will refer to this as the predicate split): In unconditionals, communication verbs but not epistemic verbs license what-value readings, as seen by the contrast between (5a) and (5b). The puzzle is summarized by the table in (7).

\section{The What-value Reading Gap}

\begin{tabular}{|l|l|c|c|}
\hline RC verb & context & what-value (A) & which-concept (B) \\
\hline \multirow{2}{*}{ epistemic } & attitude & $\checkmark$ & $\checkmark$ \\
\cline { 2 - 4 } & unconditional & $\#$ & $\checkmark$ \\
\hline \multirow{2}{*}{ communication } & attitude & $\checkmark$ & $\checkmark$ \\
\cline { 2 - 4 } & unconditional & $\checkmark$ & $\checkmark$ \\
\hline
\end{tabular}

1 Note that there are verbs of communication (e.g. manner verbs such as mumble/whisper/etc.) that tend not to have CQ readings; we set these aside. 
In this paper, we provide a fully compositional analysis of simple and nested CQs in unconditional sentences, which also derives the what-value reading gap. The paper is structured in the following way: in $\$ 2$, we discuss the standard approach to Heim's ambiguity in attitude contexts, and some background about unconditionals. Then, we turn to our proposal. We address the contrast between unconditionals and attitude contexts in two parts, beginning with the what-value reading gap in unconditionals in $\S 3$, and then finally move on to the contrast between epistemic and communication verbs in unconditional contexts in $\S 4$.

\section{Background}

In the following sections we review some background on the Individual Concept (IC) account of CQs, as well as the interpretation of unconditionals. In reviewing the IC account we focus especially on the standard treatment of Heim's ambiguity within this approach. We end the section by preparing the way for a question-based approach to CQs when they appear in unconditionals.

\subsection{Concealed Questions and Heim's ambiguity}

The puzzle introduced by the what-value-reading gap becomes even more intriguing when looking at the treatments of Heim's ambiguity in the literature. Here we will review Romero's (2005) influential analysis. Romero is one of the proponents of the Individual Concept Approach (IC-approach) to CQs, which was first introduced by Heim (1979) and later developed by Romero (2005, 2010) and Frana (2010). Heim (1979) suggests that definite descriptions with CQ-readings, like the underlined DP in (8), may be analyzed on a par with definite descriptions in temporally intensional contexts, like the underlined DP in (9).

(8) Clara knows the temperature in this room.

(9) The temperature in this room is rising.

While in (9), the temporally intensional predicate is rising forces us to look at temperature values at earlier and later (temporal) indices, the epistemic predicate know in (8) forces us to compare temperature values at different (world) indices (the actual world $\mathrm{w}$ and the worlds according to Clara's beliefs in w). Given the Montagovian treatment of sentences like (9) in terms of individual concepts (Montague 1973), Heim suggests that an analogous analysis could be given for (8). Roughly speaking, knowing an IC construed as a function from possible worlds to individuals amounts to the following: if I know the individual concept $f$ in $w_{0}$, then $f$ yields the same value at $w_{0}$ and at the worlds compatible with what I believe in $w_{0}$. 
Building on Heim 1979, Romero (2005) develops a detailed analysis of DP-CQs embedded under epistemic know. According to Romero, a parsimonious analysis of concealed question DPs follows by assuming that predicates like epistemic know are defined cross-categorially to combine both with questions (10), and individual concepts (19), (here $\operatorname{Dox}_{x}(w)$ stands for the set of worlds compatible with what the attitude holder $\mathrm{x}$ believes in world w, i.e. the set of $\mathrm{x}$ 's doxastic alternatives to $\mathrm{w}$ ). ${ }^{2}$

$$
\begin{aligned}
& \llbracket \text { know }_{\mathbf{Q}} \rrbracket^{w}=\lambda Q_{\langle s\langle s t\rangle\rangle} \cdot \lambda x_{e} \cdot \forall w^{\prime} \in \operatorname{Dox}_{x}(w):\left[Q\left(w^{\prime}\right)=Q(w)\right] \\
& \llbracket \text { know }_{\mathbf{i c}} \rrbracket^{w}=\lambda f_{\langle s e\rangle} \cdot \lambda x_{e} \cdot \forall w^{\prime} \in \operatorname{Dox}_{x}(w):\left[f\left(w^{\prime}\right)=f(w)\right] \quad \text { Romero (2005) }
\end{aligned}
$$

In the case of simple CQs, know $w_{i c}$ combines with the intension of the DP, i.e. an individual concept derived as shown in (12). ${ }^{3}$ The sample truth-conditions for a simple CQ-sentence are given in (13) below.

$$
\begin{aligned}
& \text { 【the price of milk }{ }_{i c} \rrbracket^{w}=\lambda w^{\prime} \cdot \llbracket \text { the price of milk } \rrbracket^{w^{\prime}}= \\
& \lambda w^{\prime} \cdot\left[l x_{e} \cdot x \text { is the milk price in } w^{\prime}\right]
\end{aligned}
$$

$\llbracket$ Miles knows ic $_{\text {ic }}$ the price of milk $\rrbracket^{w}=$

$\forall w^{\prime} \in \operatorname{Dox}_{\mathrm{M}}(w):\left[l x_{e} . x\right.$ is the milk price in $\left.w^{\prime}\right]=\left[l x_{e} . x\right.$ is the milk price in $\left.w\right]$

Having seen how simple CQs work in the IC-approach, we can now return to Heim's ambiguity in (3). What is special about this example is that the DP-CQ contains a CQ-embedding predicate, i.e. it is a nested CQ. Because of this, the complex DP the price that Miles knows ends up denoting an individual concept, as shown in (14) below. Given that know ${ }_{i c}$ requires an individual concept as its first argument, the trace $t_{i}$ must be of semantic type $\langle\mathrm{se}\rangle . \lambda$ abstraction at the CPlevel creates a predicate of individual concepts, which needs to intersect with the denotation of price. Thus, price must also denote a predicate of individual concepts, which we are going to derive with Nathan's IC-shifter, as shown in (15). ${ }^{4}$ (We will refer to the denotation in (15) as 'price-concept' for simplicity.)

2 At a technical level (10) does not work with the treatment of questions we later adopt, being in the Groenendijk \& Stokhof 1984 framework, but it can be straightforwardly adapted so that it does. We use this entry here for simplicity of presentation.

3 Here, we follow Frana (2010) and assume that such argument is derived by Intensional Functional Application (c.f. Heim \& Kratzer (1998)).

4 Nathan's IC-shifter ensures that the individual concepts in the extension of price are individuated via the noun's internal argument. Each price-concept so derived will be of the form the price of $x$, i.e. a function mapping every world in its domain to the price of $\mathrm{x}$ at that world (see Nathan 2006; Frana 2010 for discussion on why Nathan's type shifter is necessary to ascribe correct truth-conditions to sentences involving quantification over concepts). 


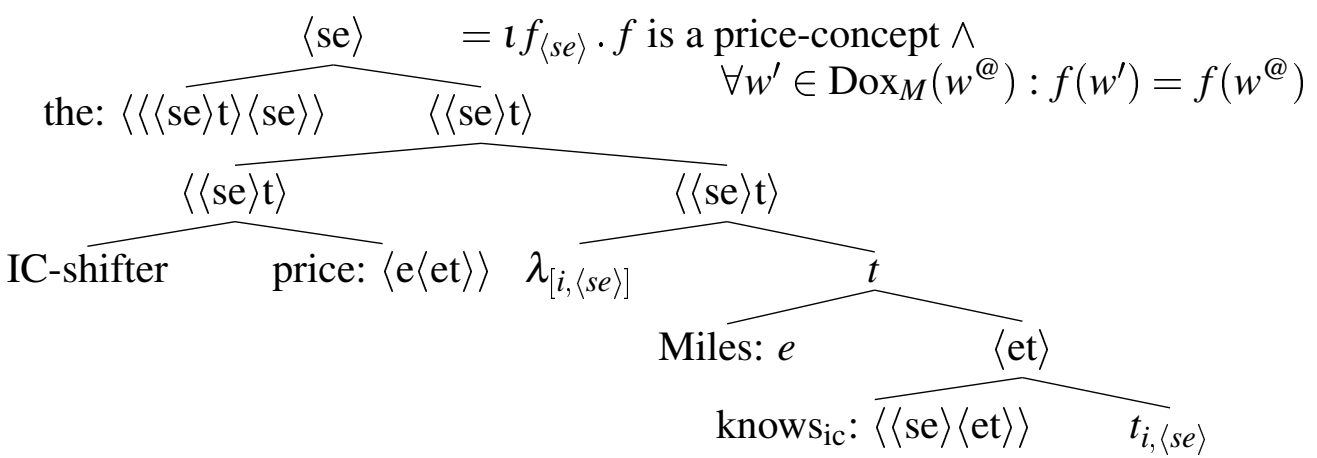

$\llbracket$ IC(price) $\rrbracket^{w}=\lambda f_{\langle s e\rangle} \cdot \exists x_{e}: \forall w^{\prime}: f\left(w^{\prime}\right)$ is the price of $x$ at $w^{\prime}$ (Nathan 2006)

Since the nested CQ here already denotes an individual concept, it can combine with know $w_{i c}$ directly, without any further intensionalization, as shown in (16) below.

$\llbracket$ Clara knows $_{\mathbf{i c}}$ the price that Miles knows $\mathbf{k i c}^{w} \rrbracket^{w}=\mathrm{T}$ iff $\llbracket \mathbf{k n o w}_{\mathbf{i c}} \rrbracket^{w}\left(\llbracket\right.$ the price that Miles knows $\left.\mathbf{i c}_{\mathbf{i c}} \rrbracket^{w}\right)=$

$$
\forall w^{\prime} \in \operatorname{Dox}_{\mathrm{C}}(w):\left[\begin{array}{l}
\llbracket \text { the price that Miles knows } \\
\mathbf{i c} \rrbracket^{w}\left(w^{\prime}\right) \\
=\llbracket \text { the price that Miles knows } \\
\mathbf{i c} \rrbracket^{w}(w)
\end{array}\right]
$$

In our context, the unique price-concept whose value is known to Miles is the individual concept that maps every world to the price of the new iPhone in that world. Let's call this concept iPHONE-PRICE. Given this context, the above formula is equivalent to (17), i.e. a what-value reading:

$$
\forall w^{\prime} \in \operatorname{Dox}_{\mathrm{C}}(w):\left[\operatorname{iPHONE}-\operatorname{PRICE}\left(w^{\prime}\right)=\operatorname{iPHONE}-\operatorname{PRICE}(w)\right]
$$

Romero's analysis reflects the intuition that the what-value reading is in some sense analogous to simple CQ-readings. In both cases, we are comparing the value of an individual concept across possible worlds (the actual world and the attitude holder's belief worlds); the difference lies in the way this individual concept comes about. In the case of simple CQs, the IC argument is provided by taking the intension of an individual denoting DP; whereas in the case of nested CQ, the DP itself already denotes an individual concept, which can be fed to know $w_{i c}$ directly.

For the which-concept reading, Romero spells out a proposal originally sketched by Heim (1979). The main idea is that in which-concept readings, we are comparing the values of a more complex concept, namely the function that maps every possible world $w^{*}$ to the concept whose value Miles knows in $w^{*}$. This higher order concept is derived by taking the intension of (14), as shown in (18).

$$
\begin{aligned}
& \llbracket \text { the price that M. knows }\langle s\langle s e\rangle\rangle \rrbracket^{w}=\lambda w^{*} \cdot \llbracket \text { the price that M. knows } \rrbracket^{w^{*}}= \\
& \lambda w^{*} \cdot\left[l f_{\langle s e\rangle} \cdot f \text { is a price-concept } \wedge \forall w^{\prime \prime} \in \operatorname{Dox}_{M}\left(w^{*}\right): f\left(w^{\prime \prime}\right)=f\left(w^{*}\right)\right]
\end{aligned}
$$


However, this function cannot be fed to know $\mathrm{w}_{\mathrm{ic}}$. In order to accomodate higherorder concepts, Romero ends up advocating for a cross-categorial denotation for CQ-embedding know, given in (19) below.

$$
\begin{aligned}
& \llbracket \text { know }_{\mathbf{C Q}} \rrbracket^{w}=\lambda f_{\langle\tau\rangle} \cdot \lambda x_{e} . \forall w^{\prime} \in \operatorname{Dox}_{x}(w):\left[f\left(w^{\prime}\right)=f(w)\right] \\
& \text { where } \tau=\langle s e\rangle \text { or }\langle s\langle s e\rangle\rangle \text { or... }
\end{aligned}
$$

(Romero 2005)

The right truth-conditions for the which-concept reading are derived by assuming that the matrix know selects for a concept of type $\langle\mathrm{s}\langle\mathrm{se}\rangle\rangle$, while the lower know inside the RC selects for a simple individual concept.

$$
\begin{aligned}
& \llbracket \text { know }_{\mathbf{C Q}} \rrbracket^{w}\left(\lambda w^{*} \llbracket \text { the price that Miles knows } \mathbf{C Q} \rrbracket^{w^{*}}\right)= \\
& \forall w^{\prime} \in \operatorname{Dox}_{\mathrm{C}}(w):\left[\begin{array}{r}
\llbracket \text { the price that Miles knows } \mathbf{C Q} \rrbracket^{w^{\prime}} \\
=\llbracket \text { the price that Miles knows } \mathbf{C Q} \rrbracket^{w}
\end{array}\right]
\end{aligned}
$$

With 'iPHONE-PRICE' again referring to the unique price-concept whose value is known to Miles at the evaluation world $w$, the formula above reduces to (21):

$$
\begin{aligned}
& \forall w^{\prime} \in \operatorname{Dox}_{\mathrm{C}}(w): \\
& \left(\begin{array}{l}
{\left[\boldsymbol{l} f_{\langle s e\rangle} \cdot f \text { is a price-concept and } \forall w^{\prime \prime} \in \operatorname{Dox}_{M}\left(w^{\prime}\right): f\left(w^{\prime \prime}\right)=f\left(w^{\prime}\right)\right]} \\
=\text { iPHONE-PRICE }
\end{array}\right)
\end{aligned}
$$

According to the formula above, (3) is true iff the unique price concept whose value Miles knows in Clara's belief worlds is the concept he actually knows, i.e. the price of the iPhone. These T-conditions correctly capture the which-concept reading.

In light of the Individual Concept analysis of Heim's ambiguity, the what-value reading gap discussed in $\$ 1$ becomes even more mysterious. On the one hand, there is the surprising fact that simple CQs are licensed in unconditionals, even though nested CQs (with epistemic predicates) cannot have a what-value reading. This fact breaks the parallelism built into Romero's account between simple CQs and what-value readings. On the other hand, it is surprising that the supposedly simpler what-value reading is absent in unconditionals, while the more complex which-concept reading is present. In Romero's account the two readings are quite intertwined and it is hard to see how one could block the what-value reading, while allowing for the which-concept reading.

\subsection{Unconditionals}

We adopt here Rawlins' (2008) analysis of unconditionals. The basic idea is that an unconditional involves quantifying over a set of exhaustive alternatives provided compositionally by the adjunct; each alternative provides a domain restriction for a main-clause operator, illustrated pictorially in (22) and more formally in (23). 
In English, the alternative set is present because the adjunct ( $\alpha$ below) is question denoting (and, Rawlins argues, has interrogative syntax). Unconditionals contrast with the case of an if-conditional in this framework (Lewis 1975; Kratzer 1981 etc.), which are not question-denoting and provide a single, non-exhaustive domain restriction. The compositional details of Rawlins' analysis beyond (23) will not be necessary for present purposes. 5

(22) No matter what disease Miles has, he should stay home from school.

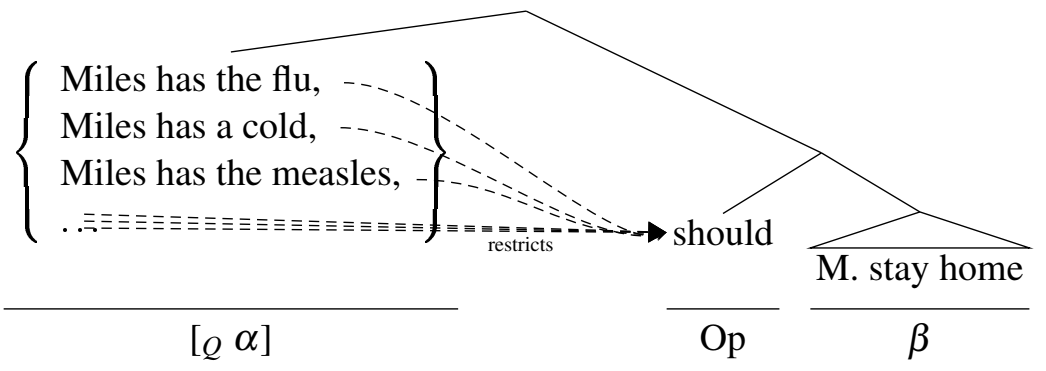

$\llbracket$ No matter $[Q \alpha]$, Op $[\beta] \rrbracket^{w}=\forall p \in \llbracket\left[\left[_{Q} \alpha\right] \rrbracket^{w}: \llbracket \mathbf{O p} \rrbracket^{w}(p)\left(\llbracket \beta \rrbracket^{w}\right)\right.$

An example like (22) can be paraphrased as: for every salient disease that Miles might have, if he has that disease, he should stay home from school. (E.g. in all deontically/bouletically ideal worlds where he has that disease, he stays home.) An alternative paraphrase involves a sequence of if-conditionals: if he has the flu, he should stay home from school, and if he has a cold, he should stay home, etc.

Before expanding this idea to CQs, it is helpful to briefly consider the selection restrictions of headed unconditionals. The generalization is that they take interrogative clauses of all types, as well as just those DPs that have a CQ interpretation. ${ }^{6}$ (The generalization is the same for no matter and regardless of adjuncts.)

$\checkmark\{$ No matter / Regardless of $\}$ who goes to the party, it will be fun.

wh-interrogative $\checkmark$

$\checkmark$ No matter whether Miles goes to the party, it will be fun. whether-interrogative $\checkmark$

5 One important point we will abstract away from is that the propositional alternatives quantified over in this construction are subject to domain restriction and we don't actually quantify over every single possible answer to the adjunct question; see Rawlins 2008.

6 There is a related but idiomatic construction in English in which a no matter clause takes that-clauses and acts sententially (does not adjoin). see Rawlins (2008) §2.1.7 for discussion. 
This data provides a piece of evidence that has been mostly lacking in the concealed question literature (see e.g. Nathan 2006; Percus 2010 for a discussion of this point): an environment where CQs pattern with questions, and nothing else. These selectional facts can be taken as evidence that concealed questions in unconditionals should be treated as questions, semantically. Additionally, Rawlins' analysis leads us to expect that the content of a headed unconditional is alternativedenoting, again pressuring an analysis of unconditional CQs towards a question denotation in at least this case. On the basis of the aforementioned evidence, i.e. (i) selectional facts, (ii) compatibility with existing analysis of unconditionals (Rawlins 2008) and (iii) the asymmetry between epistemic verbs and unconditional adverbs (the what-value reading gap), we conclude that unconditional CQs do not denote individual concepts. Rather, we propose an account where unconditional CQs are question-denoting, and receive an analysis where they introduce Hamblin alternatives (or an analog) into semantic composition. Notice though that although we argue for a question-based analysis of unconditional CQs, we do not advocate that such approach must be extended to CQs in general, or that the individual concept approach should be abandoned. Rather, we will develop a hybrid account where both individual concept and question denotations are employed for CQs in different environments.

The idea that CQs are questions is not new. Early approaches took a syntactic/transformational view of this idea (Baker 1968), but this approach is widely agreed to be non-viable and we will not review the arguments against the syntactic treatment (see Grimshaw 1979; Nathan 2006; Frana 2010 a.o.). The consensus is that if CQs are questions, it is at the level of semantic representation. Question-based approaches have seen a recent revival (Aloni 2008; Aloni \& Roelofsen 2009; Percus 2010); these approaches involve substantially different analyses and we will save a full comparison to our hybrid account for a later date. The common thread is that definite descriptions can be type-shifted into e.g. sets of propositions (question denotations in the Hamblin framework) in order to save composition.

\section{Unconditional CQs}

\subsection{The $\mathbf{Q}_{e q}$-shifter and simple unconditional CQs}

The technical puzzle then is how definite descriptions can be treated as questiondenoting in the context of unconditionals. Of the prior approaches we most closely follow Aloni (2008) (see also Roelofsen \& Aloni (2008)) in that the type-shifter takes an individual-denoting expression, and builds a copular question out of it. Our proposal is given in (29):

$$
\llbracket \mathbf{Q}_{e q}(\alpha) \rrbracket^{w}=\lambda p_{\langle s t\rangle} \cdot \exists y \in D_{\tau}: p=\left(\lambda w^{\prime} \cdot y=\llbracket \alpha \rrbracket^{w^{\prime}}\right)
$$

(Where $\tau$ is e or some intension of e) 
This type-shifter is novel in producing only equative questions. A question of this form asks what thing does $\alpha$ equal? where the two entities being equated must have the same semantic type $\tau$. In other words, the shifter forces us to compare either an individual (at an index) to another individual, or a concept (at an index) to another concept. (In particular, it will never produce specificational questions in the sense of Romero (2005); A specificational question in this sense is one where an object of type $\langle\mathrm{se}\rangle$ is compared to an object of type $\langle\mathrm{e}\rangle$, by saturation of the world argument. This will become important when dealing with Heim's ambiguity, as it will block what-value/A readings). ${ }^{7}$

We begin exemplifying the shifter with basic cases such as (31) (repeated from (2)) or (30), unconditionals with simple CQs.

(30) Regardless of the price of that iMac, you should buy it.

(31) Regardless of the computer that Clara buys, she can install LATEX.

The DPs in question denote individuals, though their value is index-dependent, so they instantiate the case of the shifter in (29) where $\tau=e$. The questions will therefore ask about the identity of an object.

A simple CQ with a relational noun (RN) such as the one in (30) works straightforwardly. The RN is saturated by its internal argument, and combines with the definite article as a simple property; the result is illustrated in (32).

(32) $\llbracket$ the price of that iMac $\rrbracket^{w}=\imath x_{e} . x$ is the price-value of that iMac in $w$

$$
\begin{aligned}
& \llbracket \mathbf{Q}_{e q}(\text { the price of that iMac }) \rrbracket^{w}= \\
& \lambda p_{\langle s t\rangle} \cdot \exists y \in D_{e}: p=\left(\lambda w^{\prime} \cdot y=\left(l x_{e} \cdot x \text { is the price-value of that iMac in } w^{\prime}\right)\right)
\end{aligned}
$$

The shifter then takes the individual-denoting expression in (32) and generates the set of alternative propositions characterized by (33), each proposition corresponding to a different possible price-value. These alternatives will have a form like, e.g. the price of the iMac is $\$ 1000$, the price of the iMac is $\$ 800$, etc. This alternative set plugs directly into the scheme for unconditional interpretation in (23), generating the truth-conditions in (34) (with $w$ as the world of evalution):

$$
\begin{array}{r}
\forall p \in\left\{p \mid \exists y \in D_{e}: p=\left(\lambda w^{\prime} \cdot y=\left(l x_{e} \cdot x \text { is the price of that iMac in } w^{\prime}\right)\right)\right\}: \\
\operatorname{should}_{w}(p)\left(\lambda w^{\prime} \cdot \text { you buy it in } w^{\prime}\right)
\end{array}
$$

7 A similar type shifter is also given in Nathan 2006 who, while not advocating a question approach, gives a type-shifter that overcomes many of the prior arguments against question approaches (see Nathan $2006 \$ 2.1$, and ex. 6). This shifter takes an individual concept, and generates a set of alternatives. However, Nathan's question shifter overgenerates with our data since it generates only specificational questions. 
For every alternative proposition describing the price of that iMac, in all accessible worlds where that proposition is true, I buy that iMac in that world.

The relative clause example in (2) works similarly; the derivation involves building a type $e$ denotation by the standard intersective analysis of restrictive relatives (e.g. Heim \& Kratzer 1998). The description uniquely identifies (at an index) something that is a computer and that Clara buys it, and the $\mathrm{Q}_{e q}$-shifter builds sets of propositions around this description. For example, if $a, b$ are particular computers in the domain (or computer-kinds), then the alternatives would be e.g. the computer that $\mathrm{C}$. buys is $a$, the computer that $\mathrm{C}$. buys is $b$, etc. Again, this alternative set plugs straightforwardly into the unconditional schema, as shown in (36), interpreting (31) ( $w$ here is the evaluation world for the sentence).

$$
\begin{aligned}
& \left.\llbracket \mathbf{Q}_{e q} \text { (the computer }\left[\lambda_{i} \text { that Clara buys } t_{i}\right]\right) \rrbracket^{w}= \\
& \qquad \lambda p_{\langle s t\rangle} \cdot \exists y \in D_{e}: p=\left(\lambda w^{\prime} \cdot y=\left(\imath x_{e} \cdot\left(\begin{array}{c}
x \text { is a computer in } w \\
\wedge \text { Clara buys } x \text { in } w
\end{array}\right)\right)\right) \\
& \forall p \in \llbracket(\mathbf{3 5}) \rrbracket^{w}: \operatorname{can}_{w}(p)\left(\lambda w^{\prime} . \text { C. installs LaTeX on it in } w^{\prime}\right)
\end{aligned}
$$

This account for simple definites makes a further prediction (see also Nathan 2006: $\S 2$ fn. 2 for the corresponding prediction of his type-shifter). Rigid definites should be infelicitous in unconditionals: when applied to a rigid definite the $\mathrm{Q}_{e q^{-}}$ shifter generates a singleton set containing just a tautological proposition (which reduces to $\left\{\lambda w^{\prime}\right.$. Miles $\left.\in D_{e}\right\}$ ), not a valid question meaning. The only type $e$ descriptions that are predicted to be licensed are those that are index-dependent.

\# No matter Miles, the party will be fun.

$$
\llbracket \mathbf{Q}_{e q}(\text { Miles }) \rrbracket^{w}=\lambda p_{\langle s t\rangle} \cdot \exists y \in D_{e}: p=\left(\lambda w^{\prime} \cdot y=\text { Miles }\right)
$$

With simple CQs and other definites out of the way, we turn to the more complex case of nested CQs, where the type-shifter involves higher-typed arguments.

\subsection{Nested CQs and the what-value reading gap}

On the IC account, the extension of a nested CQ at a given world is an individual concept $(\langle\mathrm{se}\rangle)$, not an individual. The result is that our proposed type-shifter behaves differently than in the simple CQ case, building an equative question about concepts, and generating a which-concept reading only. This can be seen in (38), which is the result of applying the $\mathrm{Q}_{e q}$-shifter to the nested DP-CQ the price that Miles knows (the details are from the earlier tree in (14)).

$$
\begin{aligned}
& \llbracket \mathbf{Q}_{e q} \text { (the price that Miles knows) } \rrbracket^{w} \\
& =\lambda p_{\langle s t\rangle} \cdot \exists y \in D_{\langle s e\rangle}: p=\left(\lambda w^{\prime} \cdot y=\left(l f_{\langle s e\rangle} \cdot f \text { is a price-concept } \wedge\right.\right. \\
& \left.\left.\forall w^{\prime \prime} \in \operatorname{Dox}_{M}\left(w^{\prime}\right): f\left(w^{\prime \prime}\right)=f\left(w^{\prime}\right)\right)\right)
\end{aligned}
$$


The alternatives generated in (38) characterize price concepts that Miles can correctly determine the value of (i.e. propositions of the form the price that $M$. knows is the price of milk, the price that M. knows is the price of cookies, etc). When combined with the unconditional schema, we get quantification over such alternatives:

$$
\begin{aligned}
& \llbracket \text { No matter } \mathbf{Q} \text { (the price that Miles knows), we should still watch. } \rrbracket^{w^{@}}= \\
& \forall p^{\prime} \in\left\{p \mid \begin{array}{c}
\exists y_{\langle s e\rangle}: p=\left(\lambda w^{\prime} \cdot y=\left[l f_{\langle s e\rangle} \cdot f \text { is a price-concept } \wedge\right.\right. \\
\left.\left.\forall w^{\prime \prime} \in \operatorname{Dox}_{M}\left(w^{\prime}\right): f\left(w^{\prime \prime}\right)=f\left(w^{\prime}\right)\right]\right)
\end{array}\right\}: \\
& \operatorname{should}_{w^{@}}\left(p^{\prime}\right)\left(\lambda w^{\prime} \text {. we still watch } w^{\prime}\right)
\end{aligned}
$$

Simple CQs and nested CQs thus come apart as a result of their compositional structure, with unconditional which-concept CQs quantifying over alternatives identifying concepts, not values. In (40) we have summarized both the simple RC case from (35) and the nested RC which-concept reading, pictorially. Each line in the curly braces is a propositional alternative, corresponding to a possible answer to the question generated by the type-shifted DP in question.

$$
\begin{gathered}
\mathrm{Q}_{e q} \text {-shifter case 1: } \\
\tau=e,
\end{gathered}
$$

$\alpha=$ "the computer that Clara buys"

$$
\begin{gathered}
\mathrm{Q}_{e q} \text {-shifter case 2: } \\
\tau=\langle s e\rangle,
\end{gathered}
$$

$$
\alpha=\text { "the price that Miles knows" }
$$
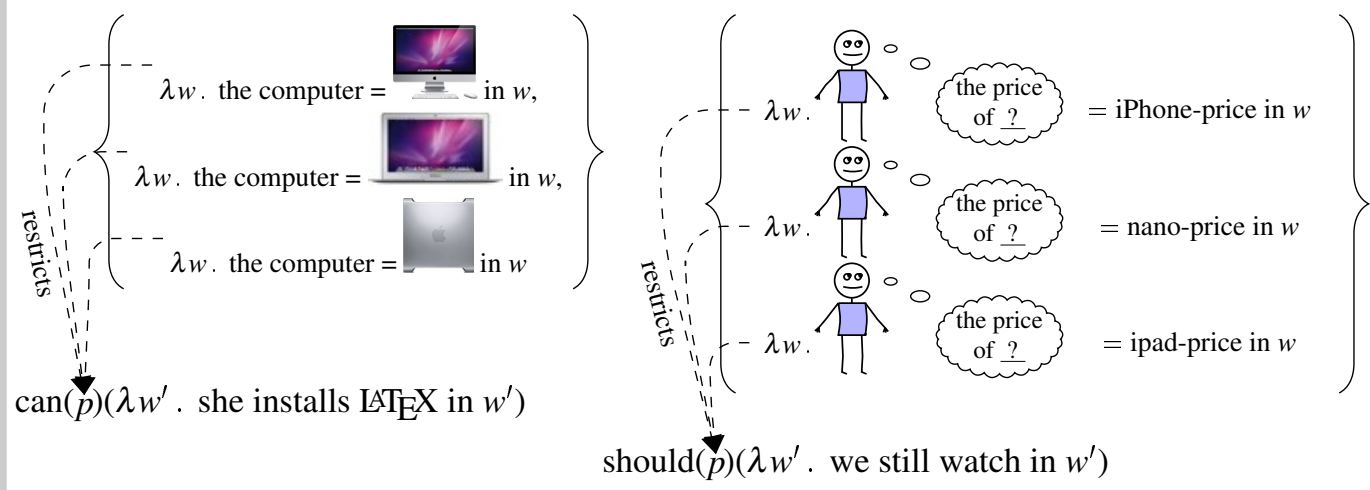

Before turning to verbs of communication in $\S 4$, we must briefly address the issue of what-value readings in attitude contexts. These cannot be generated by shifting a DP into a question, in our system. The solution lies in the hybridization of our account with Romero's. We have not eliminated individual concepts or Romero's knowic and the account of Heim's ambiguity in attitude contexts is the one already described in $\S 2.1$. To sum up, the major divide between the two types of environments - attitude vs. unconditionals - lies in the availability of a predicate selecting for individual concepts; in the case of unconditionals, there is no such predicate, and we see a 'pure' concealed question. 
Unconditional concealed questions

\section{Accounting for Predicate Split}

We now return to the contrast between epistemic predicates and communication verbs summarized in (7) - the question of why what-value readings do appear with communication verbs in unconditionals. We argue that the ambiguity displayed by unconditional CQs with communication verbs, illustrated by (6b) and (5b) is not the same type of ambiguity displayed by nested CQs in attitude contexts, but rather it is due to the fact that verbs of communication have an extra direct mention reading. This entry is in a certain sense extensional - it takes a referential argument; in our proposed analysis of unconditional CQs, this predicts a what-value reading derived in the same way as a simple CQ-RC. We proceed by first presenting some background on verba dicendi, then give evidence for the direct mention entry, and finally show the consequences for unconditional CQs and the existence of a what-value reading.

\subsection{A (simplified) semantics for Verba Dicendi}

Attitude verbs in general typically select for a range of arguments, and the standard approach is to treat the verbs as having a series of minimally different lexical entries (or one cross-categorial entry) to allow for different types of arguments. ${ }^{8}$ Thus, similarly to the standard treatment of epistemic know, as a baseline one would expect tell to have a proposition-selecting entry $\left(\right.$ tell $\left.l_{\text {that }}\right)$, a question-selecting entry $\left(\right.$ tell $\left._{Q}\right)$ and an individual concept selecting entry to allow for CQ-readings (tell ${ }_{i c}$ ).
a. Miles told me that the iPhone costs 400USD.
tell $_{\text {that }}$
b. Miles told me what the iPhone costs.
tell $_{Q}$
c. Miles told me the price of the new iPhone.
tell $_{i c}$

We begin with the standard assumption that verba dicendi have the same lexical structure as attitude predicates in general, involving quantification over some set of accessible worlds. The work of giving an account therefore involves understanding precisely which worlds to quantify over (see Davidson 1968; Ogihara 1995; Cappelen \& Lepore 1997; Kemp 2001 among many others). Roughly, we will assume someone tells someone else that $p$ just in case there is a speech event in which its agent utters something that has a denotation corresponding to $p$. There are many ways that the details could be spelled out, but for concreteness we will assume the entry in (42). To see how this works, consider how this verb might be used in the context in (43).

$$
\begin{array}{r}
\llbracket \text { tell }_{\text {that }} \rrbracket^{w}=\lambda x_{e} \cdot \lambda p_{s t} \cdot \lambda y_{e} \cdot \lambda e \cdot \exists \alpha: \text { e is an event of y uttering } \alpha \text { to } \mathrm{x} \text { at } w \\
\wedge \forall w^{\prime} \in \llbracket \alpha \rrbracket^{w^{\prime}} \rightarrow p\left(w^{\prime}\right)
\end{array}
$$

8 In the long run, we would hope for a theory of how these entries are related, i.e. an account of the regularities of the polysemy, but we will not provide one in this paper. 
(43) a. Context: Miles and Clara are having a conversation about the new iPhone, during which Miles tells her what the iPhone costs. Later on Clara reports what she has learnt from Miles by saying (41a).

b. Miles: The new iPhone just came out, it costs only 400USD. I am going to buy it tomorrow [...]

Given the lexical entry for tell $_{\text {that }}$, in (42), we (correctly) expect (41a) above to be true in the given scenario; this is because there is an utterance $\alpha$ in the given context that verifies our truth-conditions, namely the underlined sentence in (43b). The truth-conditions for the full sentence are shown in (44) below (here we are following standard assumptions regarding the interpretation of tense and aspect). ${ }^{9}$

$\llbracket$ M. told that $C$. that the iPhone costs 400USD $\rrbracket^{w}=\mathrm{T}$ iff

$\exists e . \exists \alpha:$ time $(e)<t_{0} \wedge \mathrm{e}$ is an event of Miles uttering $\alpha$ to Clara at $w$ $\wedge \forall w^{\prime} \in \llbracket \alpha \rrbracket^{w^{\prime}} \rightarrow$ the iPhone costs 400USD at $\left(w^{\prime}\right)$

So far, this is roughly the standard case of indirect speech. However, there are other ways in which Clara could have reported the speech event described in (43) that have not received attention in this literature. For example, Clara could have also felicitously uttered (41c), where a DP-CQ occurs as argument of tell. To account for concealed questions in reported speech, we would need to introduce a lexical entry for tell that selects for individual concepts, modeled after Romero's entry for knowic. A concrete proposal is given in (45) below, with truth-conditions for (41c) in (46).

$\llbracket$ tell $_{\mathbf{i c}} \rrbracket^{w}=\lambda x_{e} \cdot \lambda f_{\langle s e\rangle} \cdot \lambda y_{e} \cdot \lambda e . \exists \alpha:$ e is an event of y uttering $\alpha$ to $\mathrm{x}$ at $w$

$$
\wedge \forall w^{\prime} \in \llbracket \alpha \rrbracket^{w^{\prime}} \rightarrow\left[f\left(w^{\prime}\right)=f(w)\right]
$$

$\llbracket$ M. told ${ }_{\text {IC }}$ C. the price of the iPhone $\rrbracket^{w}=1$ iff

$\exists e . \exists \alpha: \operatorname{time}(e)<t_{0} \wedge \mathrm{e}$ is an event of Miles uttering $\alpha$ to Clara at $w$

$$
\wedge \forall w^{\prime} \in \llbracket \alpha \rrbracket^{w^{\prime}} \rightarrow\left(\operatorname{iPHONE}-\operatorname{PRICE}\left(w^{\prime}\right)=\operatorname{iPHONE}-\operatorname{PRICE}(w)\right)
$$

Assuming that $\alpha$ is resolved in favor of the underlined sentence in (43b) and that Miles was speaking the truth, then it would also be true that all the worlds compatible with the proposition expressed by "the iPhone costs only 400USD" are worlds in which the iPhone's price is the same as in the actual world. ${ }^{10}$

An entry along these lines appears necessary in order to account for straightforward CQs used to report speech. However, if verba dicendi had exactly the same types of entries as epistemic predicates, we expect no contrast between the two types of predicates with respect to CQ-readings on the account we have developed. More

9 The addition of an event argument here represents that tell is eventive, but know is stative. 10 Thus, our analysis correctly predicts that $t l_{I C}$ is factive whereas $t e l l_{\text {that }}$ is not. 
specifically, what-value readings would be blocked by the same machinery employed for nested CQs with epistemic predicates. We propose that the Predicate Split is explained by a distinction in available entries between communication verbs (as a class) and epistemic verbs. For tell we will call this entry tell ${ }_{D M}$ ('Direct Mention').

In the following section, we begin by providing independent motivation for this entry, demonstrating that none of the entries parallel to a verb like know are adequate to handle a range of data where a DP argument acts 'extensional'. Once the right entry is admitted on independent grounds, we show that the what-value readings simply follow from the way the direct-mention entry has to work. ${ }^{11}$

\subsection{Adjusted reports and the transparency effect}

Consider the report in (48) in the context given in (47).

(47) Context: Miles asks Clara what her ATM password is. Clara responds, "My PIN is 01060". In fact, her actual password is 01170, while 01060 is Northampton's zip code.

\section{Clara told Miles Northampton's zip code instead of her PIN.}

Although the report in (48) looks like a regular CQ-sentence, there is something peculiar about it: the description used in it (Northampton's zip code) does not supply the (concealed) question that was addressed in the speech event. That is, the description in the report is used transparently. Clara did not actually answer a question about the identity of Northampton's zip code, but rather attempted (and failed) to provide an answer to a different question, about her PIN. Nonetheless, the speaker can truthfully utter (48) in this context.

Applying the IC-taking entry for tell to (48) would not work here. In fact, assuming that $\alpha$ is the sentence uttered by Clara in response to Miles' question (My PIN is 01060), the truth-conditions in (49) below would predict the sentence to be true iff all the worlds in which Clara's PIN is 01060 are worlds in which Northampton's zip code is Northampton's actual zip code (i.e. 01060). But this is not at all the meaning of the sentence. Moreover, since there could be plenty of

11 A second piece of evidence that we do not have space to fully discuss here is the interpretation of Wh questions headed by a relational noun. With verba dicendi, what+relational noun can be used to ask about a value, but with epistemic verbs, it can't. A question like (i) can be answered with a value (e.g. \$3.22), but not (ii). (ii) would have to be answered by identifying the (concealed) question being resolved in some way - e.g. the iPhone's price, or what the price of the iPhone is. ((i) can also be answered in this way.) The behavior thus exactly parallels the split seen with unconditional CQs.

(i) What price did Miles tell you?

(ii) What price does Miles know? 
worlds in which Clara's PIN is 01060 , while Northampton's zip code is some other number, the sentence would also be false, contrary to intuitions.

$$
\begin{aligned}
& \llbracket \text { C. told } \text { IC M. Noho's zipcode } \rrbracket^{w}=1 \text { iff } \\
& \exists e . \exists \alpha: \text { time }(e)<t_{0} \wedge \text { e is an event of Carla uttering } \alpha \text { to Miles at } w \\
& \qquad \wedge \forall w^{\prime} \in \llbracket \alpha \rrbracket^{w^{\prime}} \rightarrow\left[\text { NOHO zip code }\left(w^{\prime}\right)=\text { NOHO zip code }(w)\right]
\end{aligned}
$$

Cases with epistemic verbs work differently. Suppose that someone takes Clara to be genuinely mistaken and that she has no idea of what Northampton's zip code might be. In this case, her state of mind cannot be reported with (50).

$$
\text { Clara knows Northampton's zip code (instead of her PIN.) }
$$

That is, a description cannot be used transparently with an epistemic verb, but can with a verb of communication.

\subsection{Tell $_{D M}$ and tell ${ }_{I C}$}

We have seen that tell can be used with a DP argument that admits a transparent/extensional reading and that such uses can not be accounted for by a standard IC-

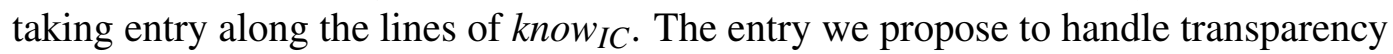
is given in (51). ${ }^{12}$ Crucially, the speech argument $s$ (the argument corresponding to the description) is used referentially, unlike in an IC-taking entry.

$$
\llbracket \text { tell }_{\mathbf{d m}} \rrbracket^{w}=\lambda x_{e} \cdot \lambda s_{e} \cdot \lambda y_{e} \cdot \lambda e \cdot \exists \alpha: \text { e is an event of y uttering } \alpha \text { to } \mathrm{x} \text { at } w
$$

$$
\wedge \forall w^{\prime}: \llbracket \alpha \rrbracket^{w^{\prime}} \rightarrow\left(s=\mathbf{f}\left(w^{\prime}\right)\right)
$$

Where $\mathbf{f} \in D_{\langle s e\rangle}$ is salient in the utterance context of $\alpha$.

According to the entry above, a speech argument of type $e$ is a suitable argument for tell, as long as it provides an answer(/value) for a salient CQ (/concept). ${ }^{13}$ This requirement is intended to explain the contrast between examples like (52) and (53). When (52) is uttered out of the blue, the sentence feels marginal. However, it improves considerably when the context of the report makes a certain concept(/question) salient to the speech event, as in (53).

$$
\text { \# John told me 400USD. }
$$

When I asked Miles for the price of the iPhone, he told me 400USD.

12 As before, the account of how polysemy in these predicates is regular is deferred for another day. For instance, one would like an explanation of why epistemic predicates don't have these entries, but verba dicendi do.

13 We leave open exactly how the value for $\mathbf{f}$ is supplied, though we suspect that it derives from a Question Under Discussion (QUD) salient in the context of the report (Roberts 1996). 
With the DM-entry above, we can account for the transparency effect data in (48). In this example, Clara used the (actual) referent of the DP Northampton's zip code in order to try to resolve a $(\mathrm{C}) \mathrm{Q}$ salient in that context (and recoverable from the report); in this case we know that she was just asked what her ATM password is, leading e.g. $\llbracket$ Clara's ATM Password $\rrbracket$ to be the salient concept. The proposed additional entry therefore explains the transparency effect, as shown in (54).

$$
\begin{aligned}
& \llbracket \text { Clara told } \mathbf{D M} \text { Miles NOHO-zip code } \rrbracket^{w}=1 \text { iff } \\
& \exists e . \exists \alpha: \operatorname{time}(e)<t_{0} \wedge \mathrm{e} \text { is an event of Clara uttering } \alpha \text { to Miles at } w \wedge \\
& \qquad \forall w^{\prime} \in \llbracket \alpha \rrbracket^{w^{\prime}} \rightarrow\left[\imath x . \text { NOHO-zip-code }(x)(w)=\imath x . \text { Clara-PIN }(x)\left(w^{\prime}\right)\right]
\end{aligned}
$$

(Where $\mathbf{f}$ is resolved as $\lambda w^{*} . \imath x$. Clara-PIN $(x)\left(w^{*}\right)$ )

The data on transparency effect provides evidence in favor of the DM-entry for $t e l l$, but it does not provide evidence for an independent $t e l l_{I C}$ entry. If the concept picked out by $\mathbf{f}$ is the one provided by the speech argument DP, the two entries yield equivalent results for simple CQs. However, we would still need tell IC $_{\text {to }}$ to account for B/which-concept readings in Heim's ambiguity in examples such as (55).

Clara told me the price that Miles told you.

We leave a full account of the relationship of these entries, and how they might be collapsed, for the future. For now we assume that the derivation of a which-concept reading with verba dicendi remains parallel to the derivation for epistemic verbs.

\subsection{What-value readings with verba dicendi are DM-readings}

Given this independently needed entry for tell, we are now in a position to return to what-value readings. The proposal is that what-value readings with verba dicendi are direct mention readings, as illustrated in (the simplified) (56). Their distribution follows from the class of embedded verb, i.e. whether that embedded verb has a direct-mention entry. Type-wise, the derivation of these readings behaves exactly like relative clause examples such as the case in (35). They involve the lowertyped instantiation of the $\mathrm{Q}_{e q}$-shifter, leading to quantification over propositions equating individuals $x$ with price-values that Miles identifies in answer to some salient price-concept $\mathbf{f}$. In the scenario described in (6), the most salient concept is iPHONE-PRICE (introduced in $\$ 2.1$ ), and so the alternatives would be propositions like "the price-value that Miles told us (as the value of iPHONE-PRICE) is \$300/, $\$ 400$, etc.".

$$
\begin{aligned}
& \llbracket \mathbf{Q}_{e q}\left(\text { the price }\left[\lambda_{i} \text { that Miles tells } \text { thM }_{D M} \mathbf{u s} t_{i}\right]\right) \rrbracket^{w}= \\
& \quad \lambda p_{\langle s t\rangle} \cdot \exists y \in D_{e}: p=\left(\lambda w^{\prime} \cdot y=\left(\imath x_{e} \cdot\left(\begin{array}{c}
x \text { is a price-value in } w \\
\wedge \text { M. tells } \\
D M
\end{array}\right)\right)\right.
\end{aligned}
$$

(where $\mathbf{f}$ is a concept salient in Miles' utterance context) 
On the other hand, epistemic know lacks an extensional entry, and consequently, the what-value reading is expected to be absent in unconditionals. ${ }^{14}$

The proposal then is that the split in behavior of unconditionals follows from a general split in behavior of communication and epistemic verbs, where the two pattern differently in extensional contexts. In consequence, what-value/A readings are possible with verba dicendi in unconditionals because these verbs have an extensional entry, generating the meaning of a simple CQ.

\section{Conclusions}

In this paper we have broadened the traditional scope of the investigation of concealed questions to include CQs in unconditionals. Our proposal is that in unconditionals, we see 'pure' CQs, necessarily derived via a type-shifter that converts individuals or individual concepts into equative questions. Crucially, CQs built this way cannot involve specificational readings in the sense of Romero (2005). The data and proposal reveals new insights into the nature of Heim's ambiguity: the ambiguity is heterogeneous with respect to verb class and derivation. In unconditional contexts, we have proposed that the presence or absence of a What-value/A reading follows from the properties of the predicates involved; verba dicendi have (independently necessary) readings that act extensional, and result in a what-value reading, but epistemic predicates do not have these readings. In attitudinal contexts, this distinction is masked by the ability of the selecting attitude predicate to interact directly with an individual concept.

We draw two major conclusions from this work. First, more fine-grained typologies of attitude predicates beyond epistemics are necessary for understanding the properties concealed questions; much future work remains to understand the nature and scope of the verb classes we have been exploring here. Second, concealed questions are not necessarily a unitary phenomenon: a hybrid individual concept / question-based approach to CQs is necessary, and may help resolve the tension between competing IC and question approaches in the recent literature.

14 Of course, know does have a semi-related extensional entry, for the so-called acquaintance reading, and we would therefore predict acquaintance unconditionals to be possible with embedded know. This is exactly right, illustrated by the most natural interpretation of (i):

(i) Regardless of the Italian city that Clara knows (best), Miles is going to Venice for his vacation. 
Unconditional concealed questions

\section{References}

Aloni, Maria. 2008. Concealed questions under cover. Grazer Philosophische Studien, special volume on Knowledge and Questions 191-216.

Aloni, Maria \& Floris Roelofsen. 2009. A pragmatic perspective on concealed questions. Manuscript.

Baker, Carl Lee. 1968. Indirect questions in English: University of Illinois Ph.D. dissertation.

Cappelen, Herman \& Ernie Lepore. 1997. On an alleged connection between indirect speech and the theory of meaning. Mind \& Language 12. 278-296.

Davidson, Donald. 1968. On saying that. Synthese 19. 130-146.

Frana, Ilaria. 2006. The de re analysis of concealed questions: a unified account of definite and indefinite concealed questions. In M. Gibson \& J. Howell (eds.), Proceedings of SALT XVI, 17-34. Ithaca, New York: CLC Publications.

Frana, Ilaria. 2010. Concealed questions: in search of answers: University of Massachusetts at Amherst Ph.D. dissertation.

Grimshaw, Jane. 1979. Complement selection and the lexicon. Linguistic Inquiry 10(2). 279-326.

Groenendijk, Jeroen \& Martin Stokhof. 1984. Studies in the semantics of questions and the pragmatics of answers: University of Amsterdam PhD dissertation.

Heim, Irene. 1979. Concealed questions. In Rainer Bäurle, Urs Egli \& Arnim von Stechow (eds.), Semantics from different points of view, 51-60. Berlin: Springer.

Heim, Irene \& Angelika Kratzer. 1998. Semantics in generative grammar. Oxford: Wiley-Blackwell.

Kemp, Gary. 2001. Samesaying, propositions and radical interpretation. Ratio 14(2). 131-152.

Kratzer, Angelika. 1981. The notional category of modality. In Hans-Jürgen Eikmeyer \& Hannes Rieser (eds.), Words, worlds, and contexts: New approaches in world semantics, 38-74. Berlin: Walter de Gruyter.

Lewis, David. 1975. Adverbs of quantification. In Edward L. Keenan (ed.), Formal semantics of natural language, 3-15. Cambridge: Cambridge University Press.

Montague, Richard. 1973. The proper treatment of quantification in ordinary English. In Jaakko Hintikka, Patrick Suppes \& J.M.E. Moravcsik (eds.), Approaches to natural language, 221-242. Dordrecht: Reidel.

Nathan, Lance. 2006. On the interpretation of concealed questions: Massachusetts Institute of Technology Ph.D. dissertation.

Ogihara, Toshiyuki. 1995. Double access sentences and references to states. Natural Language Semantics 3. 177-210.

Percus, Orin. 2010. Uncovering the concealed question (and some shifty types). Handout from SALT 20. 
Rawlins, Kyle. 2008. (Un)conditionals: an investigation in the syntax and semantics of conditional structures: UC Santa Cruz Ph.D. dissertation.

Roberts, Craige. 1996. Information structure in discourse: Towards an integrated formal theory of pragmatics. In OSUWPL volume 49: Papers in semantics, The Ohio State University Department of Linguistics.

Roelofsen, Floris \& Maria Aloni. 2008. Perspectives on concealed questions. In Proceedings of SALT XVIII, 601-618. Ithaca, New York: CLC Publications.

Romero, Maribel. 2005. Concealed questions and specificational subjects. Linguistics and Philosophy 28. 687-737.

Romero, Maribel. 2010. Concealed questions with quantifiers. In M. Aloni, H. Bastiaanse, T. de Jager \& K. Schulz (eds.), Logic, language and meaning, 21-31. Berlin: Springer.

Schwager, Magdalena. 2008. Keeping prices low: an answer to a concealed question. In Gronn Atle (ed.), Proceedings of SuB 12, 582-596. Oslo: ILOS.

Ilaria Frana

Sprachwissenschaftliches Seminar

Käte Hamburger Weg 3

37073, Göttingen

ifrana@gwdg.de
Kyle Rawlins

Room 237 Krieger Hall 3400 North Charles Street

Baltimore, MD 21218

rawlins@cogsci.jhu.edu 